\title{
A single but prolonged and eventually fatal episode of capillary leak syndrome with a diagnosis of a small tumor mass B cell lymphoma
}

\author{
Marjorie Schlier, Murielle Rondeau-Lutz, ${ }^{*}$ Jean-Christophe Weber
}

Department of Internal Medicine, Nouvel Hôpital Civil, Hôpitaux universitaires de Strasbourg, 1, place de l'hôpital, 67091 Strasbourg cedex, France.

\begin{abstract}
Accepted on
February 29th, 2014

DOI Name

http://dx.doi.org/10.3126/jaim.v3i1.10702

\section{Keywords}

lymphoma, large B-cell, diffuse; capillary leak

syndrome; paraneoplastic syndrome; monoclonal gammopathy, edema.

\section{Citation}

Marjorie Schlier, Murielle Rondeau-Lutz, JeanChristophe Weber. A single but prolonged and eventually fatal episodeof capillary leak syndrome with a diagnosis of a small tumor mass $B$ cell lymphoma. Journal of Advances in Internal Medicine 2014;03(01):34-37.
\end{abstract}

\begin{abstract}
The capillary leak syndrome is a rare and serious condition. It is characterized by acute episodes of extravasation of liquids and proteins through the capillary wall to the interstitial space, with generalized edema, hemoconcentration, hypoproteinemia. Most often, capillary leak syndrome is idiopathic. Some cases are associated with neoplastic diseases. We describe a new case of capillary leak syndrome as a paraneoplastic syndrome of a large-B-cell lymphoma. This case is unusual because of the evolution of the capillary leak syndrome during a single but extended and fatal episode. The etiologic diagnosis in this case was particularly difficult to prove because of the small tumour mass. Our case report is thus the fourth reported case of capillary leak syndrome revealing a B-cell lymphoma with a small tumour mass.
\end{abstract}

\section{INTRODUCTION}

The capillary leak syndrome (CLS) was first described in 1960 by Clarkson. ${ }^{1}$ It is characterized by the extravasation of liquids and proteins, from $200 \mathrm{KDa}$ to $900 \mathrm{kDa}$, through the capillary wall to the interstitial space. The diagnosis is clinical and biological. The process occurs episodically, with periodic and transient oedema, associated with hypotension that can lead to hypovolemic shock, haemoconcentration, and hypoalbuminaemia without proteinuria. In more than $90 \%$ of cases, immunological investigations reveal a paraproteinaemia. The end of the episodes may cause acute pulmonary oedema by the return of the fluid into the intravascular space.

Capillary leak syndrome is a rare condition. Approximately one hundred cases have been reported in the literature. Majority have been idiopathic (named Clarkson's disease). The secondary cases are often precipitated by drugs. Some cases have been associated with myeloma, lymphoma or autoimmune disease. We describe a new case of CLS as a paraneoplastic syndrome of a large-B-cell lymphoma. This case is unusual because of the evolution of the CLS during a single but extended and fatal episode. The etiologic diagnosis in this case was particularly difficult to prove because of the small tumour mass.

\section{CASE REPORT}

A 71-year-old man presented with a few weeks history of abdominal pain and an increased weight of four kilos in a few days. His significant past medical history included ischaemic heart disease and hypertension. Physical examination revealed diffuse subcutaneous oedema, a low blood pressure and a right pleural effusion. Laboratory data revealed hypoalbuminaemia $(28 \mathrm{~g} / \mathrm{l})$, thrombocytopenia $\left(110000 / \mathrm{mm}^{3}\right)$ and lymphocytopenia $\left(830 / \mathrm{mm}^{3}\right)$, elevated haematocrit (52\%) and an inflammatory response (C reactive protein $67 \mathrm{mg} / \mathrm{l}$ ).

Usual causes of oedema were excluded (hepatocellular dysfunction, cardiac failure and nephrotic syndrome). A diagnosis of CLS was considered because of the consistent laboratory results despite the lack of serum monoclonal paraprotein. The paraneoplastic origin was immediately suspected because of the lymphocytopenia associated with an elevated LDH count (1813 U/I) and the level of beta-2- microglobulin $(5,33 \mathrm{mg} / \mathrm{l})$. However, confirmation

\footnotetext{
* Corresponding author

Murielle Rondeau-Lutz,

MD, Department of InternalMedicine

Nouvel Hôpital Civil, Hôpitaux universitaires de Strasbourg, 1,

place de l'hôpital, 67091 Strasbourg cedex, France.

E-mail: murielle.rondeau@chru-strasbourg.fr
} 
of the diagnosis was difficult. There was no superficial lymphadenopathy or hepatosplenomegaly. The CT scan confirmed the presence of a pleural effusion and significant subcutaneous oedema, and demonstrated evidence of pericardial and peritoneal effusions, but did not identify any lymphadenopathy. Results of analysis of the bone marrow biopsy and the pleural fluid were normal. A PET-CT scan showed a carbohydrate hypermetabolism for several not enlarged lymph nodes situated in the retroperitoneal and mediastinal spaces and also for the first thoracic vertebra. The biopsy of this vertebra was negative for lymphoma. A transcutaneous biopsy of an abdominal lymph node was attempted. It was technically difficult due to the small size of the lymph node. The Tumor Necrosis Factor (TNF) a level was high $(9.3 \mathrm{pg} / \mathrm{ml}$, normal range 0 to $5 \mathrm{pg} / \mathrm{ml}$ ) whereas the level of Interleukin (IL) 2 was within the normal ranges $(3.2 \mathrm{pg} / \mathrm{ml}$, normal range 0 to $5 \mathrm{pg} / \mathrm{ml}$ ).

Meanwhile, despite treatment with theophylline $(400 \mathrm{mg} /$ day), oral corticosteroids $(0,7 \mathrm{mg} / \mathrm{kg} /$ day) and intravenous macromolecules fluids, the patient's condition worsened. The pleural effusion increased in size, the oedema got worse and blood pressure became unstable. Intravenous immunoglobins $(0.4 \mathrm{~g} / \mathrm{kg} /$ day for five days) were infused without any effects. After a month of hospitalization, the patient's kidney function deteriorated (creatinine clearance of $22 \mathrm{ml} / \mathrm{min}$ but probably overestimated because of oedema), he developed a disseminated intravascular coagulation and became drowsy. He was then admitted to an intensive care unit.

Twelve hours after his admission to intensive care unit, the patient died because of a massive capillary leak. The histological confirmation of the large-B-cell lymphoma from the biopsy of the retroperitoneal lymph node arrived only after the death.

\section{DISCUSSION}

This case supports the hypothesis of CLS being a paraneoplastic syndrome. The majority of described CLS cases are idiopathic but there are some documented cases of secondary CLS. Most common are iatrogenic, due to chemotherapy (IL2), granulocyte colony-stimulating factor, gemcitabine, interferon, paclitaxel) or due to medications such as sirolimus or retinoids. Some cases of CLS appear with a systemic disease, such as lupus, ${ }^{2}$ or skin diseases..$^{3,4}$ There are also several cases concomitant with potentially paraneoplastic syndrome, ${ }^{3}$ or malignant tumor. It can relate to a solid tumour such as breast adenocarcinoma.

Several cases of lymphoma which have been associated with or revealed by a CLS have been reported in the literature.
These can be related to T cell lymphomas, ${ }^{5,6}$ but are more frequently secondary to $B$ cell lymphoma, ${ }^{7-9}$ not always with a monoclonal gammopathy. One case report is an observation of an intravascular lymphoma. ${ }^{10}$ There is also one case of multiple myeloma, ${ }^{11}$ one myeloma culminating with $\mathrm{AL}$ amyloidosis, ${ }^{12}$ and a CLS with a plasma cell leukaemia. ${ }^{13} \mathrm{An}$ association between Hodgkin's disease and CLS has also been reported. ${ }^{14}$ There may be a link between idiopathic CLS and some pathology of the B-cell line which could be illustrated with the presence of a monoclonal gammopathy in most cases. It is therefore not so surprising to find secondary CLS associated with B-cell lymphoma.

The pathophysiology of CLS is still unclear at present. Several hypotheses have been proposed: endothelial damage by cytokines, ${ }^{15}$ secretion of leukotrienes, ${ }^{12}$ direct role of the paraprotein in the monoclonal gammopathies, ${ }^{16}$ but none have yet been confirmed by experimental studies.

Concerning the paraneoplastic CLS, two explanations were proposed: the responsibility of the VEGF (Vascular endothelial growth factor) and a "cytokine storm". It has been reported that numerous tumour cell lines secrete the VEGF which favours the neo-angiogenesis of tumors and which also increases the vascular permeability. Senger et al. ${ }^{17}$ studied tumor cell lines in vitro and suggested that the expression of this factor is due to the neoplastic transformation. Lesterhuis et al. measured the value of VEGF in two cases of patient with CLS and found a high level. ${ }^{18}$ This level increased during the crisis and decreased with its resolution, and was clearly high compared with a group control. The alternative explanation is more frequently discussed- the overproduction of cytokines by the tumour cells provokes a "cytokine storm". Several authors assume the hypothesis of an excessive production of IL-2. ${ }^{4,9}$ Another hypothesis is that the malignant cells could secrete TNF $\alpha$, which would cause the increase of the permeability. ${ }^{6}$ In our case, the level of the TNF $\alpha$ was increased and the level of IL-2 was normal. However these theories remain uncertain and sometimes contradictory.

The CLS often reveals the lymphoma. ${ }^{6,7,9,10,13,14,19}$ In our case, the disparity between the seriousness of the clinical scenario compared with the small size the lymphoma is surprising. Indeed, it proved difficult to confirm the diagnosis of lymphoma. Moreb et al. described the same difficulty to diagnose lymphoma for 2 CLS cases: it was about high-grade B-cell lymphomas of serous membranes without any tumor masses. ${ }^{19}$ In our case, the examination of the pleural liquid did not show cytological anomaly. In Zlotnick's case-report, ${ }^{10}$ the diagnosis of intravascular B-cell lymphoma was only made on autopsy. Our case report is thus the 4th reported case of 
CLS revealing a B-cell lymphoma with a small tumour mass. As autopsy has not been carried out hence the possibility of an intravascular lymphoma cannot be excluded.

The idiopathic CLS is characterized by episodic and acute attacks. ${ }^{20}$ In our case, the evolution extended over one month, ending in the death of the patient. Most of the case reports of CLS revealing a lymphoma have a prolonged course, $6,7,10,14,19,21$ until the death of the patient or until the beginning of an effective chemotherapy which sometimes gives an outstanding result regarding the CLS. In this regard, Ghosh's observation $^{13}$ is important because the patient had two attacks which resolved after treatment of cardiac decompensation, but it is noteworthy that the patient also received aminophylline, which has been used to treat CLS (see below). It was only after the third episode, six months later, that the diagnosis of plasma cell leukemia was made. Because of the limited analysis after the first attacks, it is possible that the plasmacytic pathology was not present from the beginning and thus there was an associated self-limiting CLS episode. It is notable that in Amoura's case series of idiopathic CLS, two out of 13 cases on progressed towards a myeloma within five years. On the contrary, a few cases of chronic forms of CLS have been described where there was no involvement of any lymphomatous disease. ${ }^{21,23,24}$

The treatment of the acute attacks relies on the replacement of fluid to maintain blood pressure and the use of vasopressors agents. However the administration of fluid has to be done carefully because of the risk of fluid overload and pulmonary oedema during the recovery phase. ${ }^{20}$ No known curative treatment is available for the CLS, therefore the treatment remains empirical. Terbutaline has been trialled because theoretically the beta-mimetics could reduce the capillary permeability due to the actions of histamine and bradykininon the endothelial contraction. The conclusions concerning the efficacy of this treatment remain unclear and conflicting. ${ }^{22}$ Some authors suggest that the increased capillary permeability could be associated with apoptotic damage to the endothelial cells. Factors increasing the intracellular cyclic AMP could preserve against apoptotic lesions. This theory can explain the use of theophylline (inhibition of the cyclic-AMP's

\section{REFERENCES}

1. Clarkson B, Thompson D, Horwith M, et al. Cyclical edema and shock due to increased capillary permeability. Am J Med 1960;29:193 216.

http://dx.doi.org/10.1016/0002-9343(60)90018-8

2. Ferard-Sasson G, Lambert M, Queyrel V, et al. Hyperperméabilité capillaire au cours du lupus systémique sans deficit congénital en inhibiteur degradation by the inhibitory activity of phosphodiesterase) and of terbutaline (increased cyclic-AMP's production) but, again, the clinical efficacy remains unclear. ${ }^{22}$ Corticosteroids have failed to show any improvement in most studies, but the doses may have been too low. Plasmapheresis has been trialled in CLS associated with a monoclonal gammopathy in order to induce removal of the paraprotein. Intravenous immunoglobulin have also been tried. ${ }^{12,25}$ All these numerous treatment have been used with varying degrees of success. ${ }^{22}$

In our observation, theophylline, corticosteroids and intravenous immunoglobulin were unsuccessful. Intravenous immunoglobulin infusion is tricky in a hypovolemic situation. Indeed, for our patient, anuria even followed this infusion.

Identifying and treating the cause is probably the most effective way to manage CLS. Chemotherapy improves most cases associated with lymphoma. $5,6,7,9,14$ The rapid improvement with chemotherapy indicates that the tumoral lysis does not exacerbate the CLS but may even improve it. The pathogenic agent doesn't seem to be present in the tumoral cells. Another hypothesis explaining the rapid efficiency of chemotherapy is that it includes high doses of corticosteroids. In our case as in the one of Moreb et al, the lymphoma's discovery and the beginning of chemotherapy have been too late. ${ }^{19}$

Conclusion: Based on this observation and the data from the literature, the following systematic approach to a patient with CLS with a prolonged evolution can be proposed: investigations into possible cancer and more specifically a lymphoma should be rapidly initiated. The possibility of an atypical presentation should be considered when investigating, eg a small tumoral mass, a lymphoma of serous membranes without any tumor masses or intravascular lymphoma. These timely investigations are important because chemotherapy seems to be the only treatment likely to improve the patient's prognosis.

\section{ACKNOWLEDGEMENT}

Many thanks to Katie Gordon for her careful read-through.

de la C1 estérase: deux nouveaux cas. Rev Med Interne2001;22(S4):532.

http://dx.doi.org/10.1016/S0248-8663(01)80232-0

3. Carsuzaa F, Pierre C, Morand JJ, et al. Capillary leak syndrome disclosing Ofuji's papuloerythroderma. Ann Dermatol Vénéréol 1996;123:559 62.

4. Dereure O, Portales P, Clot J, et al. Biclonal Sézary syndrome with capillary leak syndrome. Dermatol Basel 
Switz 1994;188:152 6.

http://dx.doi.org/10.1159/000247123

5. Capesius C, Crickx B, Belmagoug N, et al. Edematous syndrome disclosing T-cell lymphoma. Ann Dermatol Vénéréol 1992;119:882 3.

6. Takimoto Y, Imanaka F, Sasaki N,et al. Gamma/delta T cell lymphoma presenting in the subcutaneous tissue and small intestine in a patient with capillary leak syndrome. Int J Hematol 1998;68:183 91.

http://dx.doi.org/10.1016/S0925-5710(98)00053-X

7. Pineau $S$, Neel A, Moreau $A$, et al. Syndrome d'hyperperméabilité capillaire révélant un lymphome non hodgkinien au cours d'un syndrome de GougerotSjögren. Rev Med Interne 2010;31(S1):87 8.

http://dx.doi.org/10.1016/j.revmed.2010.03.096

8. Jillella AP, Day DS, Severson K, et al. Non-Hodgkin's lymphoma presenting as anasarca: probably mediated by tumor necrosis factor alpha (TNF-alpha). Leuk Lymphoma 2000;38:419 22. http://dx.doi.org/10.3109/10428190009087034

9. Andrès $E$, Vinzio $S$, Ruellan $A$, et al. Syndrome d'hyperperméabilité capillaire de la face. Presse Med 2000;29:1279 81.

10. Zlotnick DM, Merrens EJ, Fingar EL, et al. A 69-year-old male presenting with hypotension and anasarca. Am J Hematol 2008;83:311 4.

http://dx.doi.org/10.1002/ajh.21124

11. Hiraoka E, Matsushima $Y$, Inomoto-Naribayashi $Y$, et al. Systemic capillary leak syndrome associated with multiple myeloma of IgG kappa type. Intern Med Tokyo Jpn 1995;34:1220 4.

http://dx.doi.org/10.2169/internalmedicine.34.1220

12. Vigneau C, Haymann J-P, Khoury N, et al. An unusual evolution of the systemic capillary leak syndrome. Nephrol Dial Transplant 2002;17:492 4.

http://dx.doi.org/10.1093/ndt/17.3.492

13. Ghosh $\mathrm{K}$, Madkaikar M, lyer $\mathrm{Y}$, et al. Systemic capillary leak syndrome preceding plasma cell leukaemia. Acta Haematol 2001;106:118 21.

http://dx.doi.org/10.1159/000046600

14. Garland T Jr, Graber SE, Stein RS. Lymphoma presenting as anasarca. South Med J 1981;74:1157 8.

http://dx.doi.org/10.1097/00007611-19810900000038

15. Baluna R, Rizo J, Gordon BE, et al. Evidence for a structural motif in toxins and interleukin-2 that may be responsible for binding to endothelial cells and initiating vascular leak syndrome. Proc NatIAcadSci USA 1999;96:3957 62.

http://dx.doi.org/10.1073/pnas.96.7.3957

16. Zhang W, Ewan PW, Lachmann PJ. The paraproteins in systemic capillary leak syndrome. Clin Exp Immunol 1993;93:424 9.

http://dx.doi.org/10.1111/j.1365-2249.1993.tb08195.x

17. Senger DR, Perruzzi CA, Feder J, et al. A highly conserved vascular permeability factor secreted by a variety of human and rodent tumor cell lines. Cancer Res 1986;46:5629 32.

18. Lesterhuis WJ, Rennings AJ, Leenders WP, et al. Vascular Endothelial Growth Factor in Systemic Capillary Leak Syndrome. Am J Med 2009;122:e5 e7.

http://dx.doi.org/10.1016/j.amjmed.2009.01.020

19. Moreb J, Duque RE, Siddiqui T, et al. High-grade B-cell lymphoma presenting as polyserosal disease. Diagnosis by flow cytometry. Cancer 1989;64:2254-8.

20. Dhir V, Arya V, Malav IC, et al. Idiopathic systemic capillary leak syndrome (SCLS): case report and systematic review of cases reported in the last 16 years. Intern Med Tokyo Jpn 2007;46:899 904.

http://dx.doi.org/10.2169/internalmedicine.46.6129

21. Stirling CM, Boulton-Jones JM, Simpson K. Progressive oedema in a 30-year-old. Lancet 1998;352:450.

http://dx.doi.org/10.1016/S0140-6736(98)05348-3

22. Amoura Z, Papo T, Ninet J, et al. Systemic capillary leak syndrome: report on 13 patients with special focus on course and treatment. Am J Med 1997;103:514 9. http://dx.doi.org/10.1016/S0002-9343(97)00272-6

23. Airaghi L, Montori D, Santambrogio L. Chronic systemic capillary leak syndrome. Report of a case and review of the literature. J Intern Med 2000;247:731 5. http://dx.doi.org/10.1046/j.1365-2796.2000.00693.x

24. Rauzy $O$, Adoue D, Arlet P. Chronic systemic capillary leak syndrome not requiring treatment? Am J Med 1998;105:360.

25. Lambert M, Launay D, Hachulla E, et al. High-dose intravenous immunoglobulins dramatically reverse systemic capillary leak syndrome. Crit Care Med 2008;36:2184 7.

http://dx.doi.org/10.1097/CCM.0b013e31817d7c71 